\title{
MICROSTRUCTURAL DAMAGE CHARACTERISTIC OF A LAYER-TO-LAYER THREE-DIMENSIONAL ANGLE-INTERLOCK WOVEN COMPOSITE UNDER QUASI-STATIC TENSILE LOADING
}

\author{
Qian Ma ${ }^{1 \dagger}$, Ke Wang ${ }^{1,2 \dagger *}$, Shu-Dong Wang ${ }^{1}$, Hong-Wu Chen ${ }^{1 *}$, Li-Min Jin ${ }^{3-5 *}$, Hua Liu ${ }^{1}$, Xiao Qin ${ }^{1}$ \\ 1Jiangsu Research and Development Center of the Ecological Textile Engineering and Technology, College of Textile and Clothing, Yancheng Polytechnic \\ College, Yancheng 224005, P.R. China. E-mail: ycfxchw@126.com \\ ${ }^{2}$ State Key Laboratory of Bio-Fibers and Eco-Textiles, College of Textile and Clothing, Qingdao University, Qingdao 266071, P.R. China. E-mail: yc- \\ fywk@126.com \\ ${ }^{3}$ Shanghai Institute of Applied Physics, Chinese Academy of Sciences, Shanghai 201203, P.R. China. E-mail: jinlimin033@126.com \\ ${ }^{4}$ Hubei Key Laboratory of Advanced Textile Materials \& Application, Wuhan Textile University, Wuhan 430200, P.R. China. \\ ${ }^{5}$ College of Textiles, Donghua University, Shanghai 201620, P.R. China. \\ ${ }^{*}$ Corresponding author \\ †These authors contributed equally to this work.
}

\begin{abstract}
:
Three-dimensional angle-interlock woven composites (3DAWCs) are widely used for their excellent mechanical properties. The most significant feature is the existence of the undulated warp yarns along the thickness direction, which makes it interesting to study the mechanical properties in the warp direction. The quasi-static tensile behavior of a layer-to-layer 3DAWC along the undulated warp direction was studied by experimental and finite element analysis (FEA) methods. Based on the experimental results, the typical failure mode involving fibers, resin, and their interfaces was found. According to the FEA results, the stress concentration effect, key structural regions, and microstructural (yarn and resin) damage mechanism were obtained, which provided effective guidance for structural optimization design of the 3DAWC with stronger tensile resistance performance. In addition, the threestep progressive failure process of the 3DAWC under quasi-static tensile load was also described at the "yarnresin" microstructural level.
\end{abstract}

\section{Keywords:}

three-dimensional angle-interlock woven composite (3DAWC), quasi-static tensile, structural failure mechanism, finite element analysis (FEA)

\section{Introduction}

Currently, three-dimensional (3D) textile structural fabrics (3DTSFs) and their reinforced composite materials have been widely used in aerospace, transportation, sports equipment, personal protection, civil engineering, and other fields, showing vigorous vitality and lofty prospects [1,2]. According to the different interweaving forms of fibers or yarns, the 3D textile structures can be divided into different forms such as weaving, knitting, braiding, and their mixing. Each has its own advantages and disadvantages, and the most suitable structure needs to be selected according to the specific engineering conditions [3-6].

For the woven structures, the 3D orthogonal and 3D angle interlock are the two typical types [7]. For the 3D angleinterlock woven structure, it has an obvious structural advantage subjected to the external loading owing to its special structural characteristics, such as anti-delamination and impact resistance performances. According to the weaving form and the number of interlaced layers, it is divided into two types, i.e., layer-to-layer interlock and through-the-thickness interlock [8-10]. However, the most prominent feature of this type of materials is the existence of the undulated warp yarns along the thickness direction. The undulated warp yarns firmly bind the different layers to give the material a pretty good overall stability. In addition, there are straight weft yarns arranged in the weft direction, which provides good performances for the material in this direction, especially the tension resistance property.

Therefore, the 3D angle-interlock woven composites (3DAWCs) which prepared by combining the 3D angleinterlock woven fabrics (3DAWFs) with the high-performance resin also have good mechanical properties, wherein the fabric is the reinforcing phase and the resin acts as the matrix phase, and the material has the overall load-carrying capacity based on the load transmission function of the resin. Compared with the pure fabric phase materials, the load resistance capacity of composites has been effectively improved. It has shown significant advantages in terms of fatigue resistance and impact resistance [7, 11-16]. For example, in the field of antifatigue engineering, it has a better fatigue resistance performance than the 3D orthogonal woven composite with the same fiber volume fraction at the same stress levels [7]. In the high-velocity impact engineering field, the mechanical property of the 3DAWC is significantly better than that of its fabric reinforcement. Herein, the straight-arranged weft yarns play a more important role and the impact energy is rapidly spread to a large area of the composite at a high stress wave velocity, which is very good for its impact resistance capacity [16]. 
In addition, the 3DAWC will be subjected to mechanical tensile load in some engineering conditions. This cumulative quasistatic tensile process causes sudden failure to the composite, which has a great impact on industrial production and safety. Recently, the research on the mechanical properties of the 3DAWC under tensile loading has been carried out, which are mainly at the macro level, including our previous research [17].

However, for the tensile mechanical properties of the 3DAWC at the microstructural (yarn and resin) level, there is no more detailed description on the structural effect and damage mechanism, and such information is critically essential for an in-depth understanding on its failure process.

In this paper, the quasi-static tensile behavior of one kind of the layer-to-layer 3DAWC is studied at the microstructural (yarn and resin) level. Both the experimental method and the finite element analysis (FEA) are employed. The damage morphology of the 3DAWC is investigated and analyzed, especially the failure process and mechanism of the material are studied at the microstructural level, thus providing an effective guidance for the structural enhancement of the 3DAWC under tensile loading.

\section{Experimental}

\subsection{Materials}

Figure 1 shows the surface and cross-sectional representations of the 3DAWF which are used in this study. This fabric was made with high-silica glass filaments. Its specifications are listed in Table 1. The sketch diagram of the 3DAWF structure is shown in Figure 2. It indicates that the straight weft yarns are hold together perfectly by the weaving of the warp yarns. Such structural feature results in a very good structural stability for the 3DAWF. For this presented fabric structure, the tightly entangled structure between the warp yarns and weft yarns extends through the entire thickness direction of the material, providing the 3DAWF with excellent structural stability and
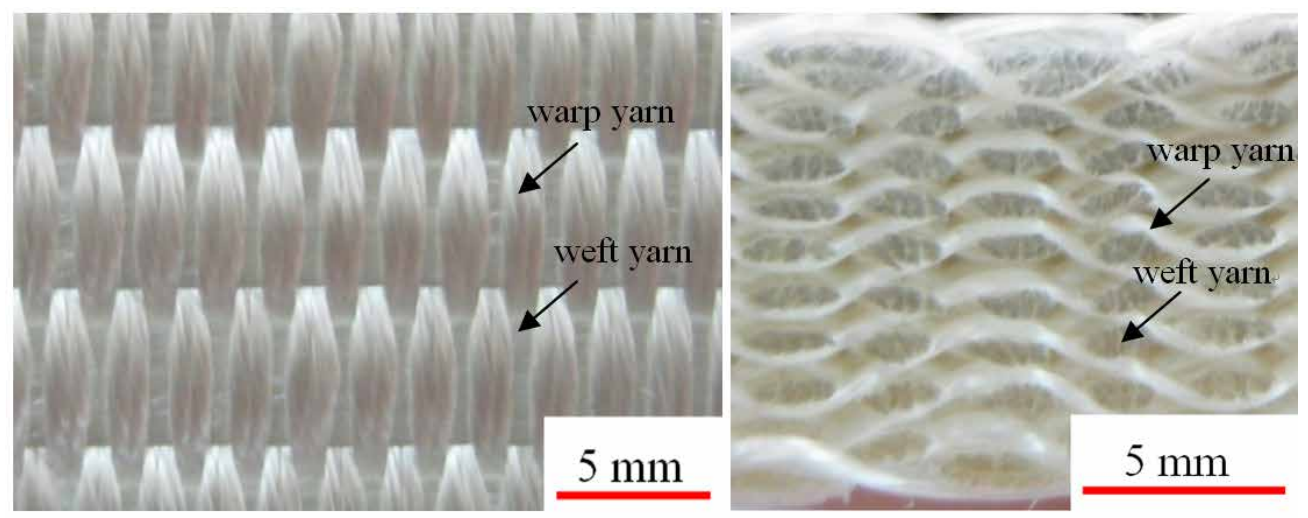

Figure 1. Photographs of the 3DAWF (3DAWF, three-dimensional angle-interlock woven fabric)

Table 1. Specifications of the 3DAWF (3DAWF, three-dimensional angle-interlock woven fabric)

\begin{tabular}{|c|c|c|c|c|}
\hline Yarns & Fiber type & Linear density (Tex) & Density (ends/cm) & Layers \\
\hline Warp & Silicon dioxide fiber tows & $440 \times 1$ & 10 & 10 \\
\hline Weft & Silicon dioxide fiber tows & $440 \times 2$ & 4 & 11 \\
\hline
\end{tabular}

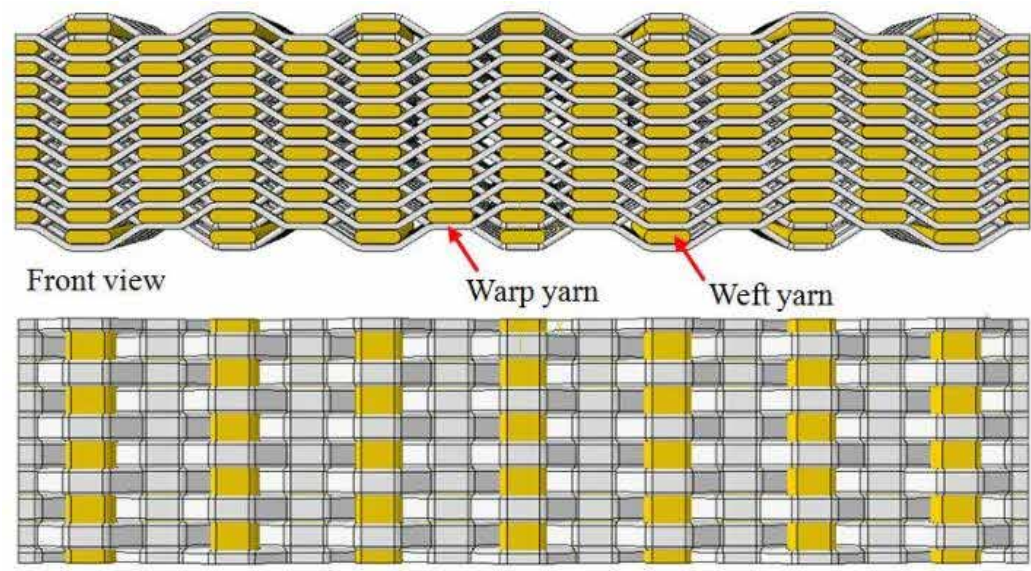

Top view
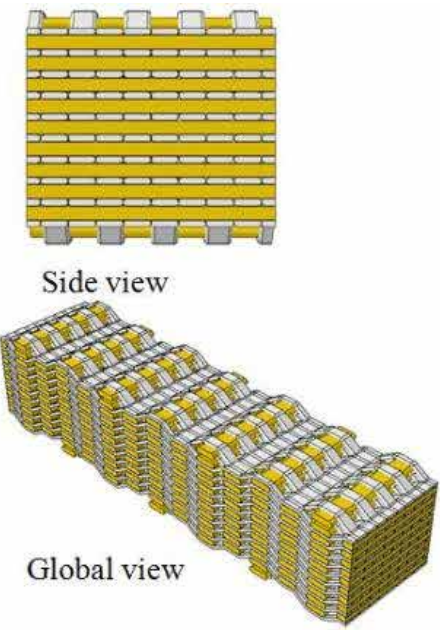

Figure 2. Sketch of the layer-to-layer angle-interlock structure 
ability to withstand external loads. When the 3DAWF is used as the reinforcement, to manufacture the 3DAWC prepared by the specific curing process with the high-performance resin, the obtained composites also have the advantages of excellent mechanical performance. In addition, when the 3DAWC is subjected to the specific external load, the entire material structure can be fully loaded by the load transfer capacity of the resin and the yarns; thus, the mechanical properties of the 3DAWC are significantly improved compared with the pure fabric reinforcement of 3DAWF.

Vacuum-assisted resin transfer molding (VARTM) technique was employed to manufacture the 3DAWC plate. The applied resin and curing agent were AROPOLTM INF 80501-50 polyester resin manufactured by Ashland Composite Polymers China and $\mathrm{AKZO}^{\circledR} \mathrm{M}-50$, respectively. Their proportion was 100:1.5 by weight. After the manufacture of the 3DAWC plate, the 3DAWC coupons presented in Figure 3 were cut with high-pressure water jet along the longitudinal and transverse directions of the composite plate. The longitudinal direction of each coupon was aligned with the warp yarns. The size (length $\times$ width $\times$ thickness) of each coupon was $200 \times 20 \times 8.4 \mathrm{~mm}^{3}$. In addition, the fiber volume fraction was approximately $54 \%$.

\subsection{Quasi-static tension tests}

As shown in Figure 4, the quasi-static tension tests of the 3DAWC coupons were conducted on an MTS 810.23 system at the speed of $2 \mathrm{~mm} / \mathrm{min}$. Aluminum tabs with the length of $50 \mathrm{~mm}$, width of $20 \mathrm{~mm}$, and thickness of $1.2 \mathrm{~mm}$ were glued at the ends of the composite coupons. The aluminum tabs will prevent the failure in the grip zones.

\subsection{FEA}

FEA was employed to figure out the stress concentration effects and critical important structural areas where prone to damage for the presented layer-to-layer 3DAWC structure under the quasi-static tensile loading condition. FEA was performed using the commercial finite element software package (ABAQUS/ Standard).

\section{Geometrical model and material parameters}

As shown in Figure 5(a), the simplified shell element FEA model of the 3DAWC was established. In addition, the material parameters of glass fiber and resin are listed in Table 2.

\section{Interaction property}

The interaction between the resin and yarns was defined as "surface-to-surface contact" with the "bonding" condition of limit bonding to slave nodes set in yarns. Moreover, the resin surface and surfaces of yarns were set as the master surface and slave surfaces, respectively.

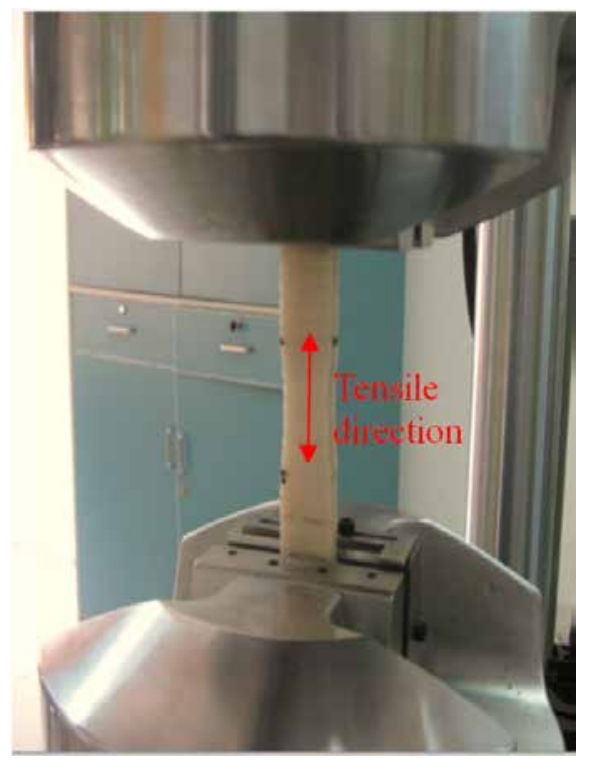

Figure 4. Experimental setup

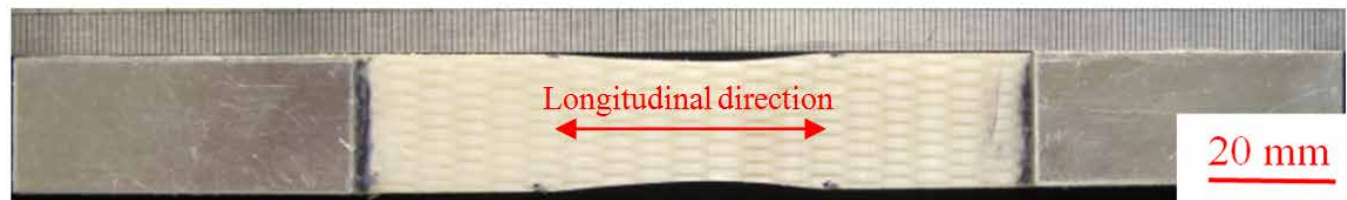

(a) Surface

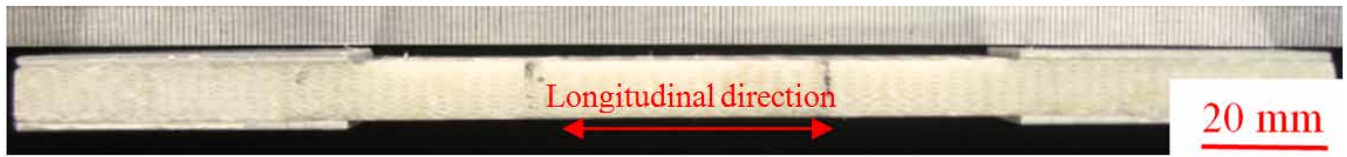

(b) Cross section

Figure 3. 3DAWC specimen for testing (3DAWC, three-dimensional angle-interlock woven composite)

Table 2. Material parameters of glass fiber and resin

\begin{tabular}{|c|c|c|c|c|c|}
\hline Materials & E (GPa) & Poisson's ratio & Density $\mathbf{( g / \mathbf { c m } ^ { 3 } )}$ & $\begin{array}{c}\text { Failure stress } \\
\mathbf{( M P a )}\end{array}$ & Failure strain $(\mathbf{\%})$ \\
\hline Glass fiber & 70 & 0.20 & 1.44 & 2,200 & 2.1 \\
\hline Resin & 3.65 & 0.35 & 1.36 & 146 & 3.2 \\
\hline
\end{tabular}




\section{Loading and boundary condition}

As shown in Figure 5(b), a displacement control of $5 \mathrm{~mm}$ as a function of time was applied at the right end of the 3DAWC structure, and the left end was fixed with no freedom of displacement or rotation. The displacement loading duration was $1 \mathrm{~s}$.

\section{Mesh}

As shown in Figure 5(c), the meshing technique of coincidence of nodes was employed. The applied element type was CPS4R Quad-dominated. Furthermore, the numbers of elements for resin, warp yarns, and weft yarns were 14,364, 8,320, and 11,880 , respectively. In addition, the total number was 34,564 .

\section{Results and discussions}

\subsection{Tensile failure morphology}

The ultimate failure morphology of the presented layer-tolayer 3DAWC under quasi-static tensile loading is presented in Figure 6, and it shows the typical damage modes of the
3DAWC. At the views of surface and cross-section, it can be found that the resin cracking, yarn breakages, and pulling out from the matrix and breakages of undulated warp yarns are the primary damage mode. In particular, the resin cracks and yarn breakages can be easily found on the surface of the sample, as well as the resin-yarn interface de-bonding is obviously observed at the cross-section.

In particular, for the fracture failure of the yarns, the damage of undulated warp yarn system can be mainly found. Taking the internal structure of the presented layer-to-layer 3DAWC and the direction of applied tension force into consideration, the length direction of each entire warp yarn parallels to the direction of the tensile load, as well as the axial direction of the weft yarns is perpendicular to the load direction. Such kind of structural feature induces the undulated warp yarns carry the majority of loads during the quasi-static tensile loading process.

The phenomenon of de-bonding is relatively easier to occur at the stress concentration regions (as indicated by red arrows in Figure 6(b)) of warp yarn-resin-weft yarn interface, where the undulated warp yarns hold the maximum amplitude of undulation.

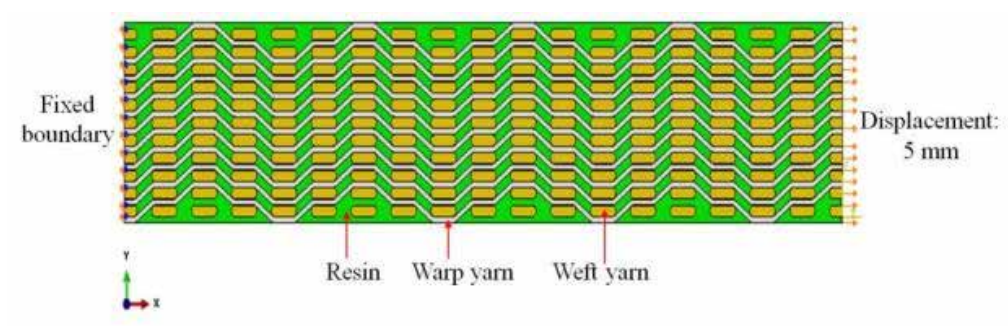

(a) Micro-structural model

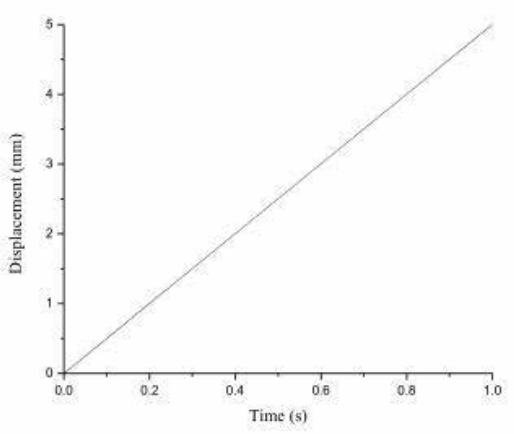

(b) Applied displacement control

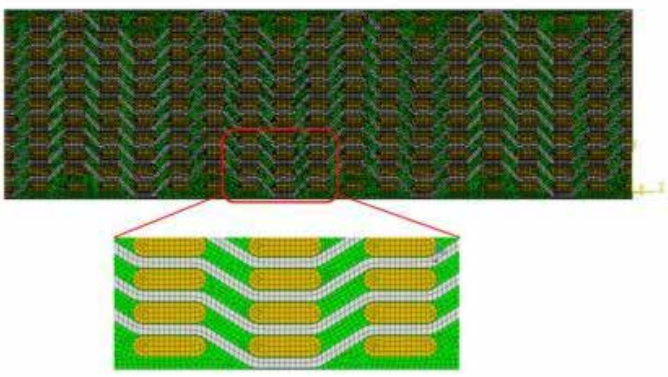

(c) Mesh (coincidence of nodes)

Figure 5. The quasi-static tension model of the 3DAWC (3DAWC, three-dimensional angle-interlock woven composite) 


\subsection{Key structural areas of stress concentration}

Figure 7 shows the FEA contour images of the structural stress distribution of the layer-to-layer 3DAWC subjected to the displacement control of $5 \mathrm{~mm}$ at different time points. As time passes, it can be seen that the high stress areas become larger due to the stress concentration effect and high volume content of yarns. Besides, it shows that the inner central region carries the majority of the applied load during the loading process, as well as the stress concentration occurs at the specific regions at warp yarns, where the maximum curvature occurs and the weft yarns cross the warp yarns. These highly stressed sites are also sites of interface de-bonding. The abovementioned phenomena are in good agreement with the experimental results which are presented in Figure 6. In addition, as shown in FEA results, the maximum stress is mainly concentrated on the undulated warp yarns, i.e., the system of undulated warp yarns along the load-carrying direction is the primary loadcarrying component for the 3DAWC under tensile loading.

\subsection{Structural failure mechanisms}

Taking the structural characteristic of the layer-to-layer 3DAWC into consideration, the phenomena summarized above should be identified as the different dominant structural damage mechanisms among three steps.

For Step I, it was mainly attributed to the resin cracking. After the tensile load was applied on the 3DAWC, at the area of finest central surface, the resin cracking began to occur after a short period of loading time. As the loading process continued, the cracking propagated along the thickness direction of the sample. Such process consumed a short period of testing time.

For Step II, the resin cracks continued to propagate into the inner layers of the composite. More importantly, the debonding at resin-yarn interface was also taken place, and simultaneously the cracks propagated from the resin to the yarns as a result of stress concentration.

In addition for Step III, it was primarily identified as the progressive breakages of yarns, especially the undulated warp yarns along the load-carrying direction. As the less and less occur of the resin damage and interface de-bonding till the ultimate saturation, with the gradual breakages of yarns. The ultimate damage of the material also occurred within a short period of time.

Based on the abovementioned analysis results, suggestions on the structural optimization design for the tensile resistance property of the layer-to-layer 3DAWC can be proposed. One of the effective methods is to reduce the bending amplitude of warp yarns as much as possible, which can be achieved by reducing the number of interlaced layers of warp yarns along the thickness direction, and for each layer in the warp direction, decrease the number of weft yarns interlaced with the warp yarns. This method not only helps to reduce the stress concentration effect but also contributes to the improvement of the tensile tolerance performance.

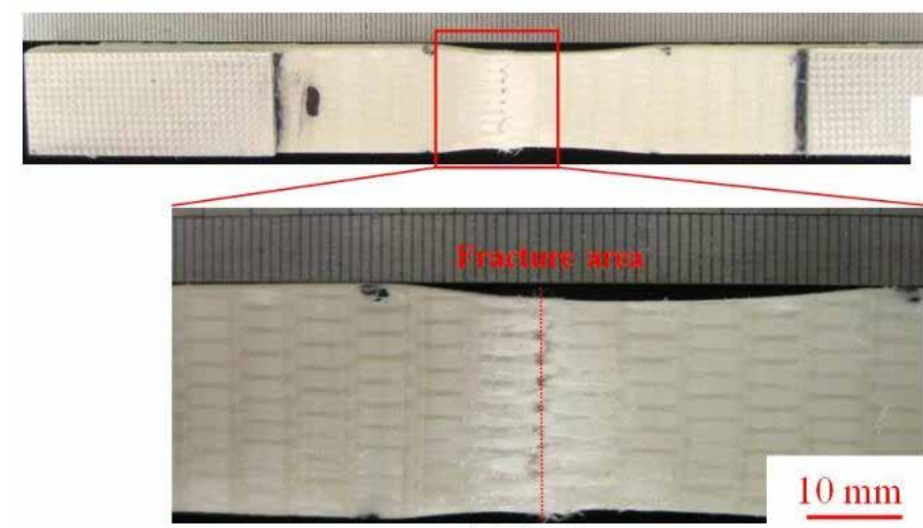

(a) Surface

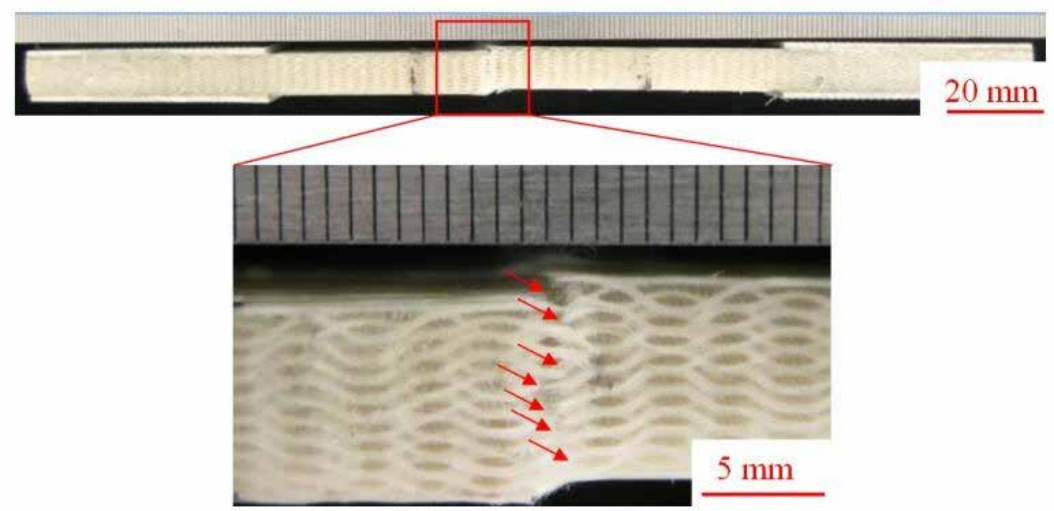

(b) Cross-section

Figure 6. Failure morphologies of the 3DAWC under quasi-static tensile loading (3DAWC, three-dimensional angle-interlock woven composite) 

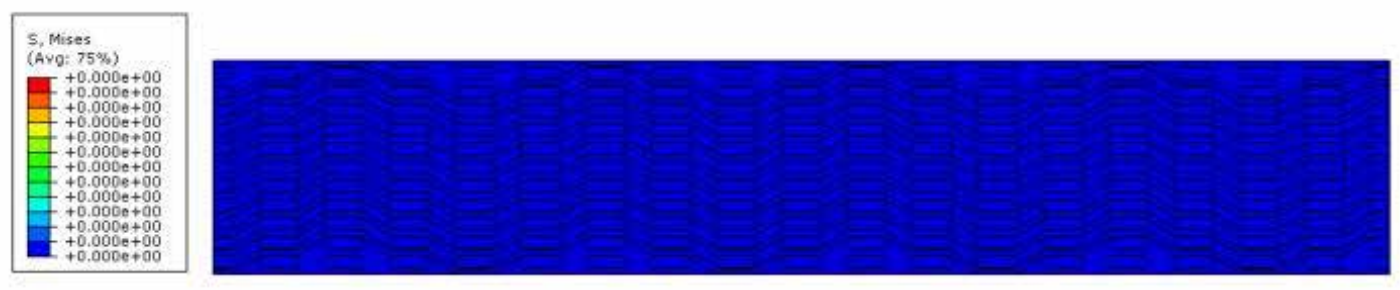

$\mathrm{T}=0 \mathrm{~s}$
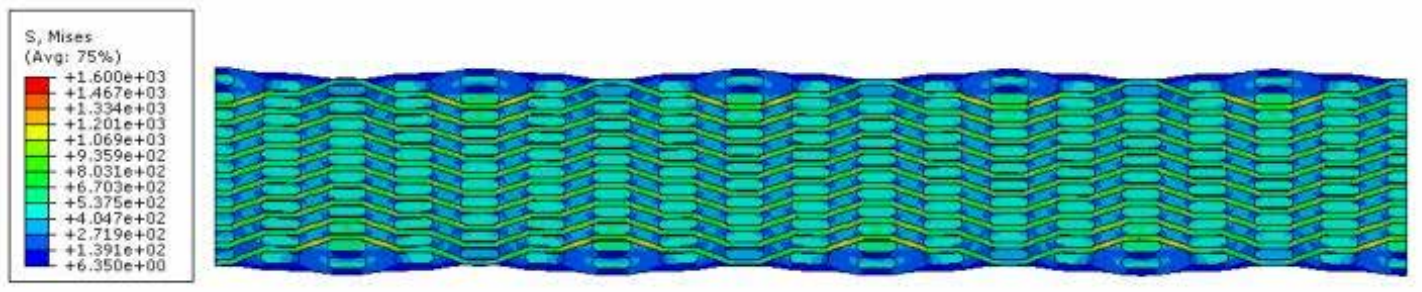

$\mathrm{T}=0.2 \mathrm{~s}$
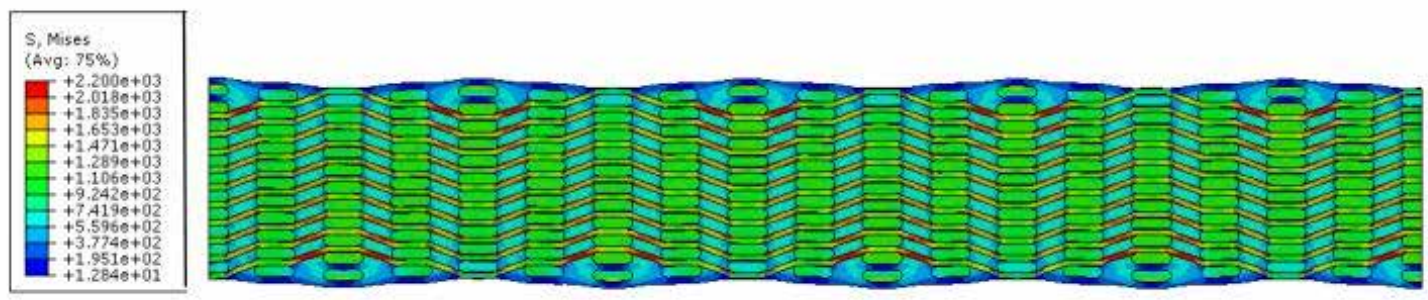

$\mathrm{T}=0.5 \mathrm{~s}$
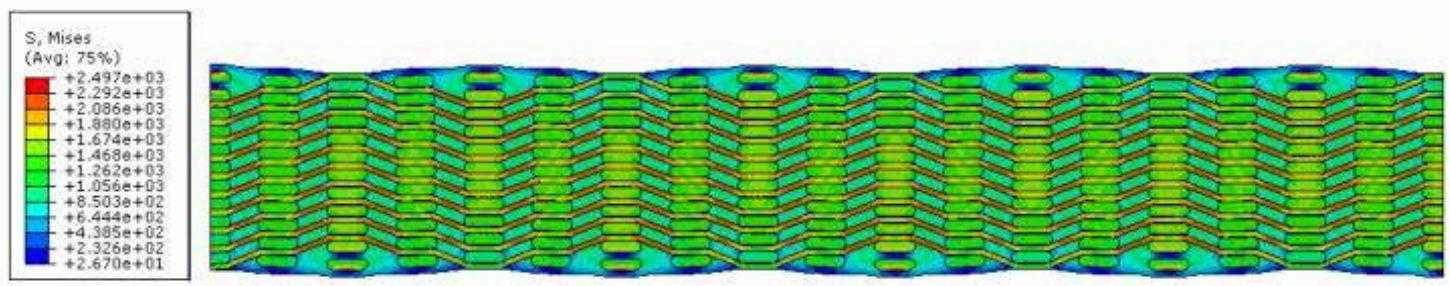

$\mathrm{T}=0.75 \mathrm{~s}$
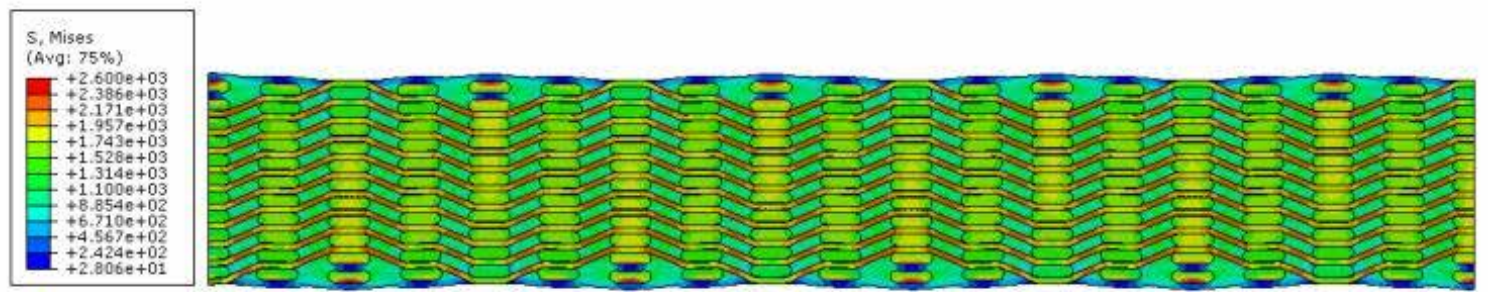

$\mathrm{T}=1 \mathrm{~s}$

Figure 7. Stress concentration regions of the 3DAWC structure at different time points (3DAWC, three-dimensional angle-interlock woven composite)

\section{Conclusions}

The quasi-static tensile failure behavior of the layer-to-layer 3DAWC along the direction of the undulated warp yarns was studied by experimental and FEA methods in this paper, and the following conclusions were obtained.

For the final failure mode of the 3DAWC, it was found that the resin cracking, the pullout and fracture of the yarns, especially the undulated warp yarns, and the de-bonding of the resinyarn interface were the main failure modes.

|Based on the FEA, the structural damage mechanism of the 3DAWC was explained at the microstructural level of the resin- yarn. It was found that the stress concentration was likely to occur at the maximum bend regions of the warp yarns. These areas were the places where resin cracking, interfacial debonding, and yarn breakage initially happened, and the damage further developed from these places to the internal part.

|The quasi-static tensile damage process of the 3DAWC can be divided into three steps, each step corresponding to a specific dominant failure mode: the first step was mainly the crack initiation and propagation of the resin, and the second step was mainly de-bonding at the resin-yarn interfaces. The third step was mainly the pullout and fracture of the yarns, especially for the undulated warp yarns, and the final failure of the material may occur in a short period of time. 
|Suggestions on the structural optimization design for the tensile resistance property of the layer-to-layer 3DAWC are proposed. One of the effective methods is to reduce the bending amplitude of warp yarns as much as possible, which can be achieved by reducing the number of interlaced layers of warp yarns along the thickness direction, and for each layer in the warp direction, decrease the number of weft yarns interlaced with the warp yarns. This method not only helps to reduce the stress concentration effect but also contributes to the improvement of the tensile tolerance performance.

\section{Acknowledgments}

This research was financially supported by the Open Project Program of Jiangsu R\&D Center of the Ecological Textile Engineering \& Technology, Yancheng Polytechnic College (YGKF-201711), the Open Project Program of Hubei Key Laboratory of Advanced Textile Materials \& Application, Wuhan Textile University (Fzxcl2017001). The authors also acknowledge the financial supports from Qing Lan Project, Overseas Research \& Training Program, the Natural Science Foundation (16KJB540005 and 18KJB540005), the Top-Notch Academic Programs Project (PPZY2015C254) of Jiangsu Higher Education Institutions of China, and the project by Advanced Study and Training and Practice Project for Young Teachers of Vocational Colleges of Jiangsu Province, China (2018QYSJ131 and 2018TDFY009).

\section{References}

[1] Mouritz, A. P., Bannisterb, M. K., Falzonb, P. J., Leongb, K. H. (1999). Review of applications for advanced threedimensional fibre textile composites. Composites Part $A$, 30(12), 1445-1461.

[2] Hu, J. (2008). 3-D fibrous assemblies: properties, applications and modelling of three-dimensional textile structures. Woodhead Publishing Limited (Sawston), 1-32.

[3] Sun, B. Z, Liu Y. K., Gu, B. H. (2009). A unit cell approach of finite element calculation of ballistic impact damage of 3-D orthogonal woven composite. Composites Part B, 40 (6), 552-560.

[4] Gu, B. H. (2007). A microstructure model for finite element simulation of 3-D 4-step rectangular braided composite under ballistic penetration. Philosophical Magazine, 87(30), 4643-4669.

[5] Jin, L. M, Hu, H., Sun, B. Z., Gu, B. H. (2010). A simplified microstructure model of bi-axial warp-knitted composite for ballistic impact simulation. Composites Part B, 41(5), 337353.
[6] Ma, P. B, Hu, H., Zhu, L. T., Sun, B. Z., Gu, B. H. (2011). Tensile behaviors of co-woven-knitted fabric reinforced composites under various strain rates. Journal of Composites Materials, 45(20), 2495-2506.

[7] Jin, L. M., Niu, Z. L., Jin, B. C., Sun, B. Z., Gu BH. (2012). Comparisons of static bending and fatigue damage between $3 D$ angle-interlock and $3 D$ orthogonal woven composites. Journal of Reinforced Plastics and Composites, 31(14), 935-945.

[8] Chen, X., Spola, M., Paya, J. G., Sellabona, P. M. (1999). Experimental studies on the structure and mechanical properties of multi-layer and angle-interlock woven structures. Journal of the Textile Institute, 90(1), 91-99.

[9] Tong, L., Mouritz, A. P., Bannister, M. K. (2002). 3D fibre reinforced polymer composites. Elsevier Science Ltd. (Amsterdam), 1-12.

[10] Bigaud, D., Dreano, L., Hamelin, P. (2005). Models of interactions between process, microstructure and mechanical properties of composite materials - a study of the interlock layer-to-layer braiding technique. Composites Structures, 67(1), 99-114.

[11] Tan, P., Tong, L. Y., Steven, G. P. (1999). Micromechanics models for mechanical and thermomechanical properties of $3 D$ through-the-thickness angle interlock woven composites. Composites: Part A, 30(5), 637-648.

[12] Sun, B. Z., Gu, B. H., Ding, X. (2005). Compressive behavior of 3-D angle-interlock woven fabric composites at various strain rates. Polymer Testing, 24(4), 447-454.

[13] Lapeyronnie P, Grognec PL, Binétruy C, Boussu F. (2011). Homogenization of the elastic behavior of a layer-to-layer angle-interlock composite. Composite Structures, 93(11), 2795-2807.

[14] Nehme, S., Hallal, A., Fardoun, F., Younes, R., Hagege, B., Aboura, Z., Benzeggagh, M., Chehade, F. H. (2011). Numerical/analytical methods to evaluate the mechanical behavior of interlock composites. Journal of Composite Materials, 45(16), 1699-1716.

[15] Cui, F., Sun, B. Z., Gu, B. H. (2010). Fiber inclination model for finite element analysis of three-dimensional angle interlock woven composite under ballistic penetration. Journal of Composite Materials, 45(14): 1499-1509.

[16] Ma, P. B., Jin, L. M., Wu, L. W. (2018). Experimental and numerical comparisons of ballistic impact behaviors between $3 D$ angle-interlock woven fabric and its reinforced composite. Journal of Industrial Textiles, DOI: 10.1177/1528083718754903.

[17] Ma, Q., Wang, K., Wang, S. D., Liu, H., Jin, B. C., Jin, L. M., Ma, P. B. (2017). Tensile damage mechanism of 3-D angle-interlock woven composite using acoustic emission events monitoring. Autex Research Journal, 18(1), 46-50. 